\title{
Análise dos efeitos do Pensamento Computacional nas habilidades de estudantes no ensino básico: um estudo sob a perspectiva da programação de computadores
}

\author{
Rivanilson da Silva Rodrigues ${ }^{1}$, Wilkerson de Lucena Andrade ${ }^{1}$, \\ Dalton Dario Serey Guerrero ${ }^{1}$, Lívia Maria Rodrigues Sampaio Campos ${ }^{2}$ \\ ${ }^{1}$ Laboratório de Práticas de Software - SPLab \\ Universidade Federal de Campina Grande - UFCG \\ Av. Aprígio Veloso, Bodocongó \\ Campina Grande, PB - Brasil \\ ${ }^{2}$ Laboratório de Sistemas Distribuídos - LSD \\ Universidade Federal de Campina Grande - UFCG \\ Av. Aprígio Veloso, Bodocongó \\ Campina Grande, PB - Brasil

\begin{abstract}
This paper presents a quantitative study that analyzes the effect of Computational Thinking - CT in the performance of students in basic education. The sample was composed of 103 students from Paraiba - Brazil. The CT was measured from Student's programming skills and the performance in High School was measured by the National High School Exam - ENEM. The result shows a moderate correlation between the performances of student's in ENEM and his programming skills. Futhermore, students proficient in programming have statistically better average than students without experience in all areas addressed in ENEM.
\end{abstract}

Resumo. O presente trabalho consiste em um estudo quantitativo que analisa o efeito do Pensamento Computacional $(P C)$ no desempenho de estudantes na educação básica. A amostra analisada foi composta por 103 estudantes do estado da Paraíba. O PC foi mensurado a partir do conhecimento dos estudantes em programação de computadores, já o desempenho ao final da educação básica foi medido por meio do Exame Nacional do Ensino Médio - ENEM. Os resultados indicam a existência de uma correlação moderada entre o desempenho dos alunos no ENEM e suas habilidades em programação, além disso, alunos proficientes em programação têm médias estatisticamente melhores que alunos sem experiência em todas as áreas abordadas no ENEM.

\section{Introdução}

O Pensamento Computacional - PC é um conjunto de habilidades para resolução de problemas desenvolvidas ao estudar conteúdos provenientes da Ciência da Computação [Wing 2006]. Atualmente, pesquisas em PC apresentam propostas curriculares e cursos de curta duração para o desenvolvimento ou inserção do PC no nível básico de ensino 
e acredita-se que essas habilidades podem contribuir para o desenvolvimento cognitivo de estudantes estimulando capacidades como raciocínio lógico, abstração e resolução de problemas, consideradas hoje como fundamentais para a formação básica. Contudo, em geral, não há avaliação quantitativa sobre a influência do PC na aprendizagem de outros conteúdos.

De acordo com Han Koh [Koh et al. 2014] os benefícios do PC na educação ainda não são claros, Grover e Pea [Grover and Pea 2013] acrescentam que ainda há muito a ser feito para desenvolver uma compreensão teórica e prática mais clara das competências computacionais em estudantes do ensino básico. Para os autores, antes da proposição de novos currículos para educação é necessário entender como é possível avaliar o Pensamento Computacional e compreender quais os seus efeitos no processo de aprendizagem formal.

Nesse contexto, a presente pesquisa tem por objetivo analisar o efeito do Pensamento Computacional no desempenho de estudantes na educação básica. Buscamos responder às seguintes questões de pesquisa: (RQ1) O Pensamento Computacional tem alguma relação com o desempenho dos estudantes no ensino básico? (RQ2) O Pensamento Computacional pode melhorar o desempenho dos estudantes no ensino básico? Para isso, desenvolvemos um estudo quantitativo do tipo ex post facto utilizando uma amostra fornecida por 103 estudantes de cursos relacionados à TI e Ciência da Computação no estado da Paraíba. Avaliamos o desempenho destes ao final da educação básica através das médias de cada um no Exame Nacional do Ensino Médio - ENEM em relação às habilidades em Pensamento Computacional (mensurado em termos do conhecimento em programação de computadores) de cada estudante durante o ensino básico.

Os resultados mostram que existe uma correlação moderada entre o desempenho de estudantes ao fim da educação básica e as habilidades do Pensamento Computacional desenvolvidas pela programação. Além disso, observamos que estudantes proficientes em programação possuem desempenho superior de até $13.49 \%$ em relação àqueles com pouco ou nenhum nível de experiência.

O trabalho segue a seguinte estrutura: na Seção 2, apresenta-se uma breve revisão da literatura abordando o Pensamento Computacional, o Exame Nacional do Ensino Médio (ENEM) e suas relações; a Seção 3 traz alguns trabalhos relacionados com o escopo do presente trabalho; a Seção 4 apresenta o método utilizado no desenvolvimento da pesquisa; a Seção 5 apresenta os resultados e as ameaças à validade da pesquisa e por fim, a Seção 6 apresenta as conclusões e trabalhos futuros.

\section{Fundamentação Teórica}

Para melhor compreender a natureza e importância do problema e os fundamentos da pesquisa, essa seção aborda alguns conceitos sobre o ENEM e Pensamento Computacional.

\subsection{Pensamento Computacional}

O trabalho de Papert sobre o construcionismo e a linguagem de programação LOGO foi fundamental para o desenvolvimento do Pensamento Computacional, pois apresentou o computador como instrumento capaz de apoiar a construção do conhecimento e trabalhou na hipótese de que a programação de computadores poderia facilitar o processo 
de resolução de problemas e o aprendizado em diversas áreas [Papert 1980]. Mais recentemente, Wing [Wing 2006] intensificou os debates sobre a natureza do Pensamento Computacional, sua definição e suas aplicações.

Segundo Wing, o PC envolve resolução de problemas, projeto de sistemas e o entendimento do comportamento humano através de conceitos fundamentais da Ciência da Computação [Wing 2006]. Para a Google, o PC consiste em um conjunto de habilidades e técnicas para resolução de problemas utilizadas por Engenheiros de Software no desenvolvimento de sistemas complexos. Estas envolvem principalmente conceitos de decomposição de problemas; projeto de algoritmos; reconhecimento de padrões; generalização de padrões e abstração [Google 2014].

Barr e Stephenson [Barr and Stephenson 2011] apresentam 9 conceitos que compõem o núcleo do Pensamento Computacional: Coleta de dados: Processo de coleta de dados ou informações sobre um problema; Análise de dados: Dar sentido aos dados, encontrar padrões, e tirar conclusões; Representação de dados: Representar e organizar dados em gráficos, tabelas, texto ou figuras. Decomposição de problemas: Quebrar um problema complexo em tarefas menores e gerenciáveis; Abstração: Reduzir a complexidade para definir idéias principais; Algoritmos e Procedimentos: Sequencia de passos para resolução de um problema ou atingir algum fim; Automação: Usar computadores ou máquinas para realizar tarefas repetitivas; Paralelização: Organizar recursos para, simultaneamente, realizar tarefas para alcançar um objetivo comum; Simulação: Representação ou modelo de um processo. A simulação também envolve experimentos sendo executados usando modelos.

A aquisição do PC acontece por meio do estudo de fundamentos de Ciência da Computação, podendo ou não ser integrados a disciplinas de currículos escolares para formação básica. De acordo com Resnick [Resnick et al. 2009] o estudo de programação de computadores é uma forma de compreender o núcleo de ideias relacionadas ao PC, pois pode aprimorar habilidades como raciocínio lógico, resolução de problemas e pensamento algorítmico.

\subsection{Exame Nacional do Ensino Médio - ENEM}

O ENEM tem por objetivo avaliar o desempenho dos estudantes ao fim da educação básica com o intuito de melhorar a qualidade desse nível de escolaridade. As áreas de conhecimento e componentes curriculares avaliados nesse exame são: Ciências Humanas (História, Geografia, Filosofia e Sociologia); Ciências da Natureza (Química, Física e Biologia); Linguagens e Redação (Língua Portuguesa, Literatura, Língua Estrangeira - Inglês ou Espanhol, Artes, Educação Física e Tecnologias da Informação e Comunicação); e por fim, Matemática [INEP 2015]. A Tabela 1 apresenta as competências esperadas para todas as áreas abordadas no exame.

\subsection{Relações entre o Pensamento Computacional e o ENEM}

De maneira semelhante ao PC o ENEM envolve no seu cerne a resolução de problemas. Ela está presente como critério avaliativo em todos os eixos cognitivos abordados no exame. A seguir apresentamos as relações entre as competências do ENEM (ver Tabela 1) e os conceitos do PC descritos em [Barr and Stephenson 2011]: 
Tabela 1. Matriz de Referência comum a todas as áreas de conhecimento. Fonte: Inep

\begin{tabular}{|l|l|}
\hline \multicolumn{1}{|c|}{ Competência } & \multicolumn{1}{|c|}{ Descrição } \\
\hline $\begin{array}{l}\text { Dominar lingua- } \\
\text { gens (DL) }\end{array}$ & $\begin{array}{l}\text { Dominar a norma culta da Língua Portuguesa e fazer uso das linguagens } \\
\text { matemática, artística e científica e das línguas espanhola e inglesa. }\end{array}$ \\
\hline $\begin{array}{l}\text { Compreender } \\
\text { fenômenos (CF) }\end{array}$ & $\begin{array}{l}\text { Construir e aplicar conceitos das várias áreas do conhecimento } \\
\text { para a compreensão de fenômenos naturais, de processos histórico- } \\
\text { geográficos, da produção tecnológica e das manifestações artísticas. }\end{array}$ \\
\hline $\begin{array}{l}\text { Enfrentar } \\
\text { situações- } \\
\text { problema (SP) }\end{array}$ & $\begin{array}{l}\text { Selecionar, organizar, relacionar, interpretar dados e informações re- } \\
\text { presentados de diferentes formas, para tomar decisões e enfrentar } \\
\text { situações-problema. }\end{array}$ \\
\hline $\begin{array}{l}\text { Construir } \\
\text { argumentação } \\
\text { (CA) }\end{array}$ & $\begin{array}{l}\text { Relacionar informações, representadas em diferentes formas, e co- } \\
\text { nhecimentos disponíveis em situações concretas, para construir } \\
\text { argumentação consistente. }\end{array}$ \\
\hline $\begin{array}{l}\text { Elaborar propos- } \\
\text { tas (EP) }\end{array}$ & $\begin{array}{l}\text { Recorrer aos conhecimentos desenvolvidos na escola para elaboração de } \\
\text { propostas de intervenção solidária na realidade, respeitando os valores } \\
\text { humanos e considerando a diversidade sociocultural. }\end{array}$ \\
\hline
\end{tabular}

Dominar linguagens (DL): O PC pode apoiar o desenvolvimento dessa competência uma vez que seu núcleo faz uso da linguagem matemática e científica;

Compreender fenômenos (CF): Esse processo está relacionado à obtenção de informações a partir da observação de um determinado ambiente, assim essa competência envolve claramente os conceitos de coleta, análise e representação de dados, podendo ainda envolver o conceito de simulação;

Enfrentar situações-problema (SP): Envolve principalmente os conceitos de abstração para a compreensão do problema; Coleta, análise e representação dos dados relativos ao problema; Decomposição, diminuindo a complexidade do problema e algoritmos e procedimentos para construção sistêmica de uma solução;

Construir argumentação (CA): Os critérios de coleta, análise e representação de dados estão relacionados com a construção de argumentação, tendo em vista a necessidade do levantamento de argumentos (coleta e análise de informações) a partir de um texto, imagens ou gráficos (representação de dados);

Elaborar propostas (EP): Elaborar uma proposta de intervenção ou de solução para um problema envolve Dominar linguagens (DL), Compreender fenômenos (CF), Enfrentar situações-problema (SP) e Construir argumentação (CA), de modo que pode abranger pelo menos 5 dos 9 critérios apresentados por [Barr and Stephenson 2011].

Analisando essas competências é possível perceber que conceitos relacionados ao PC estão presentes de maneira implícita na Redação, uma vez que trata da elaboração de uma proposta de intervenção para um determinado problema.

\section{Trabalhos Relacionados}

Com o objetivo de prover argumentos quantitativos para a premissa de que habilidades em computação são fundamentais para estudantes no ensino básico, Oliveira [Oliveira et al. 2014] apresenta um estudo quantitativo que correlaciona o desempenho de alunos do $6^{\circ}$ ao $9^{\circ}$ ano do Ensino Fundamental a habilidades do Pensamento Computacional. Para isso aplicou um teste de raciocínio lógico baseado em um modelo de Máquina 
de Turing proposto no seu trabalho. Como conclusões o autor expressa a existência de uma correlação positiva significante entre a avaliação realizada e o desempenho médio dos alunos após o ano letivo.

Em seu trabalho, Van Dyne [Van Dyne and Braun 2014] descreve o processo de avaliação do Pensamento Computacional em um curso para melhorar as habilidades de resolução de problemas em estudantes de Ciência da Computação com deficiência no aprendizado de matemática. Os conteúdos abordados no curso envolveram raciocínio lógico, resolução de problemas e introdução a programação. Com base em uma análise estatística ao final do trabalho a autora aponta que estudar conteúdos que abrangem o Pensamento Computacional pode melhorar as habilidades analíticas de qualquer estudante.

Os estudos realizados por Van Dyne e Oliveira [Van Dyne and Braun 2014, Oliveira et al. 2014] apresentam propostas distintas de avaliação dos efeitos do Pensamento Computacional. Nosso estudo difere de ambos principalmente do ponto de vista metodológico,uma vez que avalia o desempenho de dois grupos estudantes ao fim da educação básica, estes possuem perfis semelhantes, mas são distintos em relação ao conhecimento em programação durante a educação básica. Além disso, nosso trabalho traz evidências de disciplinas que podem ser beneficiadas com a inserção do Pensamento Computacional na educação.

Wangenheim [Wangenheim et al. 2014] apresenta a proposta de uma unidade instrucional para o ensino de computação no Ensino Fundamental de forma integrada a conteúdos de Literatura e Artes. O estudo foi desenvolvido em uma turma do primeiro ano com crianças de 6-7 anos de uma escola privada e ao final da unidade as crianças conseguiram programar uma história interativa usando o Scratch. Os dados coletados durante o experimento foram analisados de forma qualitativa e a autora concluiu que o ensino de computação pode ser integrado ao currículo da educação básica de forma harmônica e interdisciplinar.

No intuito de estimular o desenvolvimento do Pensamento Computacional de França [de França and do Amaral 2013] apresenta o design e avaliação de uma oficina de Scratch realizada com 24 estudantes de uma escola pública no Estado de Pernambuco. O objetivo da oficina foi desenvolver habilidades relacionadas ao Pensamento Computacional e avaliar a aprendizagem dos participantes em relação aos conteúdos desenvolvidos durante o curso. Ao final do seu trabalho a autora apresenta uma análise qualitativa e considera que apesar das limitações, os resultados apontam a viabilidade do desenvolvimento do Pensamento Computacional na educação básica.

Diferente de França [de França and do Amaral 2013] e Wangenheim [Wangenheim et al. 2014], que apresentam propostas de cursos e avaliações qualitativos em termos do aprendizado do Pensamento Computacional. Nosso trabalho apresenta resultados quantitativos que apoiam a hipótese de que o Pensamento Computacional é uma importante habilidade para ser desenvolvida durante o ensino básico, uma vez que pode aprimorar o desenvolvimento cognitivo de estudantes em diferentes áreas.

\section{Metodologia}

O presente trabalho tem natureza empírica sendo caracterizada como uma pesquisa do tipo ex post facto. Particularmente, investiga possíveis relações de causa e efeito entre fatores e certos aspectos pertinentes a uma população ou grupo [Cohen et al. 2011]. 


\subsection{Design da Pesquisa}

Tendo em vista o objetivo de analisar o efeito do Pensamento Computacional no desempenho de estudantes na educação básica, utilizamos as seguintes variáveis para compor o design da pesquisa expresso na Tabela 2:

Experiência em programação de computadores (ExP): Consideramos que as habilidades relacionadas à prática em programação de computadores compreendem o núcleo do Pensamento Computacional [Resnick et al. 2009].

Desempenho no ENEM (D): Consideramos também que esta variável é uma representação confiável das aptidões do estudante ao fim da educação básica.

Tabela 2. Design da pesquisa

\begin{tabular}{|c|c|c|}
\hline Grupos & Variável Independente & Variável Dependente \\
\hline Grupo A & $E x P$. & $D_{1}$ \\
\hline Grupo B & & $D_{2}$ \\
\hline
\end{tabular}

O design apresentado na Tabela 2 considera uma análise entre dois grupos de estudantes com perfis similares pertencentes a mesma população, onde o fator ExP é a única característica que distingue ambos os grupos.

\subsection{Questões de pesquisa e hipóteses}

As questões de pesquisa que motivaram o desenvolvimento desse trabalho foram:

RQ1: O Pensamento Computacional tem alguma relação com o desempenho dos estudantes no ensino básico?

H1.0: Não há evidência de correlação entre o desempenho dos estudantes ao fim da educação básica e seu nível de proficiência em Pensamento Computacional;

H1.1: Existe uma correlação entre o desempenho dos estudantes ao fim da educação básica e seu nível de proficiência em Pensamento Computacional;

RQ2: O Pensamento Computacional pode melhorar o desempenho dos estudantes no ensino básico?

H2.0: Não há diferença estatística entre as médias do ENEM de ambos os grupos;

H2.1: Estudantes pertencentes ao grupo A possuem desempenhos superiores em relação aos do grupo B;

\subsection{Coleta, análise primária dos dados e caracterização dos grupos}

A análise proposta neste estudo considerou uma amostra da população de estudantes de cursos relacionados à Ciência da Computação e TI do estado da Paraíba. Os dados para análise foram fornecidos voluntariamente por 103 estudantes matriculados em cursos nas cidades de João Pessoa, Rio Tinto, Campina Grande, Patos e Cajazeiras por meio de um formulário online. Os dados coletados foram relativos ao tipo de instituição que cada estudante frequentou durante a educação básica, sua experiência em relação à programação, mensurada através de uma escala likert, e seu desempenho detalhado de cada eixo cognitivo avaliado no ENEM. 
A coleta dos dados ocorreu entre os meses de setembro e outubro de 2014. Os dados coletados foram organizados em uma tabela e passaram por uma análise primária antes da realização de qualquer teste. $\mathrm{O}$ objetivo desta etapa foi identificar inconsistências nas respostas dos participantes, por exemplo, um respondente pode informar que aprendeu sobre uma linguagem de programação durante o ensino médio e ao mesmo tempo informar que sua escala de conhecimento sobre o tema é nula. Tais informações foram identificadas e optou-se pela eliminação dessas.

Após a coleta e análise primária dos dados, um total de 103 amostras foram consideradas para análise neste estudo. Assim, dois grupos foram caracterizados como mostra a Tabela 3:

Tabela 3. Caracterização dos grupos

\begin{tabular}{|c|c|c|c|}
\hline Grupos & Rede Pública & Rede Privada & Rede Federal \\
\hline Grupo A & 10 & 26 & 5 \\
\hline Grupo B & 24 & 35 & 3 \\
\hline
\end{tabular}

\subsection{Análise dos dados}

Para análise dos dados foi utilizada a linguagem de programação R e o software RStudio. O processo de análise dos dados envolveu o balanceamento dos grupos A e B utilizando reamostragem baseada no método bootstrap. Assim, foram formados e analisados grupos balanceados com as mesmas proporções de alunos das esferas particular, pública e federal.

Os testes de Shapiro-Wilk e de Levene foram utilizados para analisar respectivamente a normalidade dos dados e a homocedasticidade dos dados, ambos pressupostos para o teste t de Student, utilizado para análise da diferença entre as médias de cada grupo. O teste de correlação de Pearson foi utilizado para analisar a relação entre o desempenho no ENEM e as habilidades dos estudantes em programação.

\section{Resultados e discussão}

Para responder à primeira questão de pesquisa RQ1: O Pensamento Computacional tem alguma relação com o desempenho dos estudantes no ensino básico? Foi realizado o teste de correlação de Pearson entre o desempenho dos estudantes ao fim da educação básica e seu nível de proficiência em Pensamento Computacional com 95\% de confiança. A Tabela 4 apresenta o coeficiente de correlação amostral de Pearson $(r)$ para cada eixo cognitivo abordado no ENEM.

Analisando cada coeficiente fica evidente a existência de uma relação moderada positiva entre as variáveis estudadas, uma vez que para todos os casos $0.30<r<0.70$. Logo, rejeitamos a hipótese nula H1.0: Não há evidência de correlação entre o desempenho dos estudantes ao fim da educação básica e seu nível de proficiência em Pensamento Computacional; e aceitamos a hipótese alternativa H1.1: Existe uma correlação entre o desempenho dos estudantes ao fim da educação básica e seu nível de proficiência em Pensamento Computacional.

A partir dessa análise é possível inferir que existe uma relação positiva entre o Pensamento Computacional e o desempenho dos alunos na educação básica e que essa pode 
Tabela 4. Coeficiente de correlação de Pearson

\begin{tabular}{|l|c|c|c|c|c|}
\hline & Matemática & Linguagens & Humanas & Natureza & Redação \\
\hline Escala Likert & 0.3801 & 0.3899 & 0.3349 & 0.4206 & 0.4111 \\
\hline
\end{tabular}

decorrer da aptidão desses alunos em resolução de problemas, critério base na avaliação do ENEM.

Para responder à segunda questão de pesquisa RQ2: O Pensamento Computacional pode melhorar o desempenho dos estudantes no ensino básico? realizamos um teste pareado com os dados grupos A e B considerando como hipótese alternativa que as médias do grupo A são maiores que a do grupo B. Para garantir o rigor estatístico da análise os pressupostos do teste $\mathrm{t}$ (normalidade e homocedasticidade dos dados) foram avaliados. Todos os testes expressos foram realizados com nível de confiança de $95 \%$.

Para verificar a hipótese nula sobre a normalidade e homogeneidade dos dados coletados foram realizados respectivamente os testes de Shapiro-Wilk e Levene. A Tabela 5 apresenta o p-value dos testes para cada conjunto de médias do ENEM.

Tabela 5. P-value dos testes de normalidade, homocedasticidade e t-Student

\begin{tabular}{|c|c|c|c|c|c|}
\hline Teste & Matemática & Linguagens & Humanas & Natureza & Redação \\
\hline Shapiro-Wilk & 0.3091 & 0.2509 & 0.3847 & 0.07579 & 0.8807 \\
\hline Levene & 0.4038 & 0.1875 & 0.5986 & 1.0000 & 0.9614 \\
\hline t-student & 0.0026 & 0.0259 & 0.0107 & 0.0035 & 0.0060 \\
\hline
\end{tabular}

Analisando os resultados do teste Shapiro-Wilk e Levene na Tabela 5, para um nível de significância $\alpha=0.05$, constatamos que não podemos rejeitar as hipóteses de normalidade e homogeneidade, pois obtivemos um $\mathrm{p}$-value $>\alpha$ em ambos os testes para cada conjunto de dados do ENEM.

O p-value do teste t é observado na Tabela 5 e para um nível de significância $\alpha=0.05$ rejeitamos a hipótese nula H0: Não há diferença estatística nos resultados do ENEM de ambos os grupos, pois para todos os eixos cognitivos abordados no ENEM, temos um $\mathrm{p}$-value $<\alpha$. Assim, podemos assumir a hipótese alternativa H2.1: Estudantes pertencentes ao grupo A possuem desempenhos superiores em relação aos do grupo B; Tabela 6.

A diferença média entre os grupos pode ser observada de maneira detalhada na

Tabela 6. Diferença média e percentual entre os grupos

\begin{tabular}{lccccc}
\hline & Matemática & Linguagens & Humanas & Natureza & Redação \\
\hline Grupo A & 704.47 & 603.12 & 650.63 & 599.51 & 716.70 \\
Grupo B & 644.07 & 569.85 & 608.52 & 546.61 & 631.52 \\
$\begin{array}{l}\text { Diferença média } \\
\text { em pontos }\end{array}$ & 60.40 & 33.27 & 42.11 & 52.90 & 85.18 \\
\hline$\%$ & & & & & \\
\hline
\end{tabular}

Com base nos dados disponíveis na Tabela 6 verificamos que a diferença média percentual entre os grupos varia de $5.84 \%$ a $13.49 \%$. Em termos de pontuação, significa 
que estudantes com proficiência em práticas do Pensamento Computacional tiveram um aumento de 33.27 até 85.18 pontos em relação ao grupo de estudantes que não possuíam nenhum nível de conhecimento sobre o tema.

\subsection{Ameaças à Validade}

Validade de constructo: o questionário utilizado para coleta dos dados foi validado por uma equipe de especialistas. Em relação às ameaças sociais, os participantes não sabiam quais as hipóteses do trabalho, os dados referentes ao ENEM foram fornecidos voluntariamente pelos alunos.

Validade de conclusão: os pressupostos dos testes estatísticos foram seguidos, no entanto, não foi possível tratar o baixo poder estatístico uma vez que a amostra é considerada pequena. Para tratar a heterogeneidade foi feito um balanceamento das amostras de acordo com o perfil dos grupos.

Validade interna: em relação a instrumentação, o questionário foi avaliado previamente por especialistas antes da sua divulgação. A ameaça de seleção não é relevante para essa pesquisa uma vez que os estudantes envolvidos não passaram por nenhuma intervenção e não conheciam a hipótese de pesquisa.

Validade externa: não é possível generalizar os resultados uma vez que a amostra não é representativa da população de estudantes brasileiros.

\section{Conclusão}

O presente trabalho buscou analisar a relação do Pensamento Computacional com o desempenho de estudantes ao final do ensino básico. Avaliamos o desempenho no ENEM de dois grupos de estudantes, ambos pertencentes a cursos na área de Ciência da Computação do estado da Paraíba. De modo geral, cada grupo diferiu entre si apenas pela experiência em programação adquirida antes da realização do exame.

Ao final da pesquisa foi observado que existe uma correlação moderada entre o desempenho dos estudantes no ENEM e a experiencia destes em programação. Além disso, alunos com experiência em programação obtiveram um desempenho melhor em todos os eixos cognitivos abordados no exame em relação aos que não estudaram programação. Esses resultados de certa forma eram esperados, pois a matriz de referência do ENEM tem como fundamento a habilidade de resolução de problemas que também é fortemente explorada por parte do Pensamento Computacional.

Os resultados apresentados corroboram com resultados qualitativos apresentados na literatura sobre o Pensamento Computacional, além disso, as evidencias quantitativas apresentadas nesta pesquisa sugerem que o estudo e a prática de programação podem aprimorar o desempenho de alunos em diferentes contextos e áreas do conhecimento que envolvam conceitos de resolução de problemas. Contudo, tendo em vista o pequeno número de amostras analisadas ainda não é possível generalizar o resultado dessa pesquisa para outras populações de estudantes.

Como trabalho futuro, pretendemos realizar novos experimentos buscando mitigar as ameaças à validade já mencionadas, bem como responder algumas questões derivadas desse estudo: O estudo de programação é o método mais efetivo para aquisição do Pensamento Computacional? O Pensamento Computacional pode ser considerado melhor em 
CBIE-LACLO 2015

Anais do XXVI Simpósio Brasileiro de Informática na Educação (SBIE 2015)

relação a outros métodos de resolução de problemas? Como mensurar a proficiência em Pensamento Computacional?

\section{Referências}

Barr, V. and Stephenson, C. (2011). Bringing computational thinking to k-12: What is involved and what is the role of the computer science education community? ACM Inroads, 2(1):48-54.

Cohen, L., Manion, L., and Morrison, K. (2011). Research Methods in Education. Education, Research methods. Routledge.

de França, R. S. and do Amaral, H. J. C. (2013). Proposta metodológica de ensino e avaliação para o desenvolvimento do pensamento computacional com o uso do scratch. Anais do Workshop de Informática na Escola, 1(1).

Google (2014). Exploring computational thinking. Disponivel em: https://www.google.com/edu/computational-thinking/. Acesso em: $03 \mathrm{de}$ Jul 2014.

Grover, S. and Pea, R. (2013). Computational thinking in k-12 a review of the state of the field. Educational Researcher, 42(1):38-43.

INEP (2015). Sobre o enem (2015). disponivel em: http://portal.inep.gov.br/web/enem/sobre-o-enem. acesso em: 12 fev 2015.

Koh, K. H., Nickerson, H., Basawapatna, A., and Repenning, A. (2014). Early validation of computational thinking pattern analysis. In Proceedings of the 2014 Conference on Innovation \&\#38; Technology in Computer Science Education, ITiCSE '14, pages 213-218, New York, NY, USA. ACM.

Oliveira, O. L., Nicoletti, M. C., and del Val Cura, L. M. (2014). Quantitative correlation between ability to compute and student performance in a primary school. In Proceedings of the 45th ACM Technical Symposium on Computer Science Education, SIGCSE'14, pages 505-510, New York, NY, USA. ACM.

Papert, S. (1980). Mindstorms: Children, Computers, and Powerful Ideas. Basic Books, Inc., New York, NY, USA.

Resnick, M., Maloney, J., Monroy-Hernández, A., Rusk, N., Eastmond, E., Brennan, K., Millner, A., Rosenbaum, E., Silver, J., Silverman, B., and Kafai, Y. (2009). Scratch: Programming for all. Commun. ACM, 52(11):60-67.

Van Dyne, M. and Braun, J. (2014). Effectiveness of a computational thinking (cs0) course on student analytical skills. In Proceedings of the 45th ACM Technical Symposium on Computer Science Education, SIGCSE '14, pages 133-138, New York, NY, USA. ACM.

Wangenheim, C. G. v., Nunes, V. R., and Santos, G. d. (2014). Ensino de computação com scratch no ensino fundamental: Um estudo de caso. Revista Brasileira de Informática na Educação, 22(03):115.

Wing, J. M. (2006). Computational thinking. Commun. ACM, 49(3):33-35. 